\title{
中国种裳
}

\section{大白鼠中缝大核含5-羟色胺的 神经元的电生理学鉴定}

江振裕马午

(中国科学院上海生理研究所)

\section{摘要}

本文报道由逆行刺激激活的中琏大核内的缝一脊神经元，其轴突传导速度和自发 放电率变异甚大. 比较对昭动物和事先给 5,6-双羟色胺(一种专一作用于 5-羟色胺 细胞的神经毒) 的动物的缝一脊神经元的电活动, 发现在给药动物找不到传导速度慢 于 $12 \mathrm{~m} / \mathrm{s}$ 、自发放电率又低于 $3 \mathrm{~Hz}$ 的单位活动, 故具备这两特征的神经元被判别为 含 5-羟色媵的。此外, 还用锋电位间隔的变异系数来测试各单位自发放电的规则 性, 结果表明传导速度慢的单位放电比传导速度快的更不规则。

中㖓大核一直被认为在中枢镇痛机制中起重要作用 ${ }^{[1-5]}$, 然而这个神经核团仅含有少量 5-羟色胺能 (5-HT) 神经元, 而大量的却是非 5-HT 神经元 ${ }^{[6]}$, 以往许多研究工作并没有对 此加以区分. 因此, 运用电生理学方法来鉴别中缝大核内 5-HT 神经元的活动应是有趣的, 会有助于今后阐明这类神经元的生理功能. 最近 Wessendorf 等人 ${ }^{[7]}$ 报道, 用逆行刺激技术结 合使用神经毒 5,7-双羟色胺, 㨉示中缝大核 $5-\mathrm{HT}$ 神经元的轴突传导速度为 $0.7-1.0 \mathrm{~m} / \mathrm{s}$ 和 $3.1-6.0 \mathrm{~m} / \mathrm{s}$ 两类. 本项研究比较了正常的和经神经毒 5,6 -双羟色胺 $(5,6-\mathrm{DHT})$ 处理的 两组大鼠中㖓大核㖓脊神经元的活动特征, 包括轴突传导速度, 自发放电速率和规则性等, 着 眼于鉴别 5-HT 神经元.

\section{一、实验方法}

实验用去 52 只成年雄性大白鼠、系上海饲养的Wistar 种, 体重在 220--300g. 用豆拉坦 $1.1 \mathrm{~g} / \mathrm{kg}$ 体重作腹腔内注射将动物麻醉. 切开气管, 插人导管, 为接人工呼吸器用. 将左颈静 脉切开, 插入细塑导管, 以备注射肌松剂用. 动物头被固定在立体定位仪上, 在枕骨正中部钻 孔, 直径 $2 \mathrm{~mm}$, 切开硬脑膜, 在小脑表面灌注溥层琼脂盐水溶液, 以防皮层干燥并减小脑组织 的搏动. 静脉注射三碘季胺酚 $50 \mathrm{mg} / \mathrm{kg}$ 体重将动物麻瘰, 接上人工呼吸器, 行闭合式人工通 气, 参数是每分钟 80 次, 每次 $8-14 \mathrm{ml}$ 空气. 借助心电图 II 导联以监护动物一般状态, $\mathrm{R}-\mathrm{R}$ 间隔通常为 $160-200 \mathrm{~ms}$. 用一块自控的直流电加热板垫于动物体下, 使其直肠温度维持在 $37^{\circ} \mathrm{C}$ 左右. 


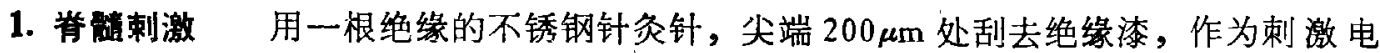
极. 无关电极则采用一个铜质环，绕在体温计顶端，置入动物直肠内．在动物胸 ${ }_{10}$ 脊椎处作椎 板切除术，切开硬脊膜。将刺激电极由外向内斜插人左侧胸檤背外侧索内 $0.5 \mathrm{~mm}$ 深处. 每 隔 $2 s$ 施加一次矩形阴极电脉冲, 波宽 $0.2 \mathrm{~ms}$, 强度变动于 $1-9 \mathrm{~mA}$ 间, 使之有效地逆行激活煡 脊神经元.

2. 微电极记录采用尖端小于 $1 \mu \mathrm{m}$ 的玻璃微电极, 充灌 $5 \% \mathrm{NaCl}$ 溶液, 内含 $2 \%$ 勏 天蓝染料, 其直流电阻在 6-12 M0. 为了寻找中㖓大核内的琏脊神经元, 将记录电极按 Palkovits 等人的脑定位图谱坐标 $(\mathrm{AP} 3.9-5.0 ; \mathrm{LO})^{[8]}$, 沿脑干正中线徐徐刺入, 观察有无被 逆行刺激激活的单位放电，直到小脑皮层下 $9 \mathrm{~mm}$ 处. 然后提起电极，向前或向后平移 $200 \mu \mathrm{m}$, 作再次穿刺, 如此不断探查. 将记录到的单位放电信号经高输入阻抗放大器放大后, 输人记忆示波器; 与此同时, 信号还通过整形, 被并联地输送到一个定时积分器, 进行笔写记 录, 和一个四道磁带录音器, 为进一步分析用.

3. 组织学检查 每次实验后, 以 $20 \mu \mathrm{A}$ 阴极电流通过记录电极 $20 \mathrm{~min}$ 。在深麻醉下处 死动物,取脑,切取脑桥一延脑段浸泡在 $10 \%$ 福尔马林液内. 5 至 7 天后作 $40 \mu \mathrm{m}$ 厚冰冻切片. 按常规进行中性红染色. 显微镜检查, 滂天蓝染色点呈圆形, 直径 $100-200 \mu \mathrm{m}$ 。通过投影, 将礿记点描绘在相应的脑干横切面图上. 凡电极穿刺针迹不在中缝大核区域内的实验，其资 料摒弃不用。

\section{二、实 验结果}

\section{1. 琏滕神经元的逆行激活}

采用 Wolstencroft ${ }^{[9]}$ 提出的三个标准来鉴别煡脊神经元:

（1）恒定的潜伏期 凡逆行激活的单位反应,不论其潜伏期的长短,总是维持在一个稳 定值,并和刺激次数无关. 本项研究观察到逆行激活的琏脊神经元反应的潜伏期变动于 1.5$70 \mathrm{~ms}$ 间, 多数为 $2.5-30 \mathrm{~ms}$. 测量了各单位的兴奋阈值. 图 1 表示一次实验记录的 35 个缝 脊单位的阈值和轴突传导速度间的相关关 系. 可以看出, 凡传导速度慢于 $20 \mathrm{~m} / \mathrm{s}$ 的单 位,其兴奋阈与传导速度呈反比关系,最高阈 值达 $9 \mathrm{~mA}$; 相反,对于超过 $20 \mathrm{~m} / \mathrm{s}$ 的单位, 则 其阈值在 I $-2 \mathrm{~mA}$ 间. 此结果提示, 本文所 用的刺激方式可以有效地兴奋背外侧索内各 类神经纤维, 不管它们距离刺激电极尖端近 或远.

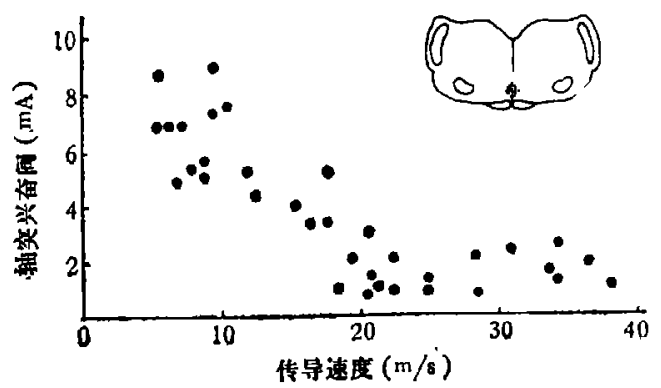

（2）跟随高频刺激的能力 由于本实 验常用大于 $5 \mathrm{~mA}$ 的刺激电流以期兴奋中煡

图 1 一次实验中 35 个被逆行激活的缝等单位们 轴突兴奋间对传导速度的关系 大核内较小的神经元, 因此常常产生由顺行激活的单位反应. 区别这点是不难做到的,因为当 用五脉冲组成的串波作刺激时, 顺行激活的单位反应跟不上高于 $20 \mathrm{~Hz}$ 的刺激频率. 还观察 到, 通过中缝大核区的过路纤维有时也能被电刺激背外侧索所激活. 在这种情况下, 单位反应 活动可跟上频率高达 $800 \mathrm{~Hz}$ 的刺激, 并且这种逆行激活的动作电位, 其升支陡直, 时程短约 
Ims. 与此相反, 琏脊神经元的逆行胞体反应可以跟随刺激频率 30-80 Hz. 并且如图 2(a) 所示，其锋电位升支有一曲折，表示始段和 胞体树突两种成分, 此外, 锋电位时程多为 1.5--2 ms, 这里应强调指出, 那些轴突传导 很慢的㖓脊单位, 锋电位时程更长, 并且升支 上常呈现突出的小丘, 这些很可能是无髓鞘 神经元的逆行胞体反应的特征.

（3）碰撞现象有时, 逆行刺激不能 产生动作电位, 这是由于该电位正巧落在前 一个自发放电的不应期内所造成的, 这就是 碰撞现象. 可是, 在中琏大核大多数神经元 的放电速率是很慢的, 所以要采用另一种方 法, 即将一个自发的单位放电放大后的信号, 同时触发示波器扫描和刺激器, 随后调节刺 激脉冲输出的时迟. 当时迟足够长时, 可以 看到由逆行刺激产生的单位反应, 可是,一旦 时迟短于一倍的逆行反应潜伏期和一个动作 电位不应期之和时, 就不再能引起逆行反应 了(图 2(b)). 这样便证明细胞外记录到的 具有自发放电的单位正是由逆行刺激激活的 揵脊神经元.
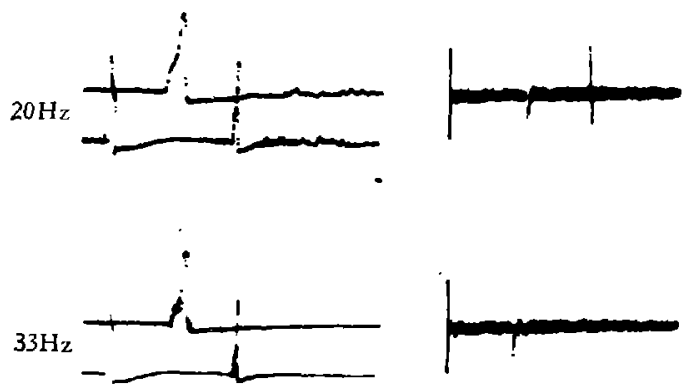

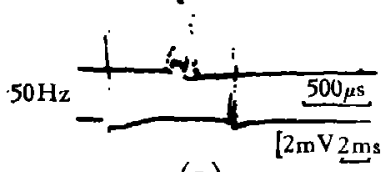

(a)

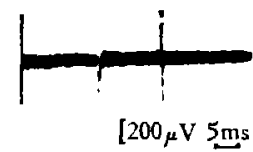

(b)

图 2(a) 一个琎脊单位对逆行串波刺激的反应 （下线系５个反应曲线的重叠记录，刺放频率标记在图旁；上 线是下线扫描的扩展, 以便观察反应诐形细节; 横线为时标， 坚线为校准电压)

(b)碰撞现象

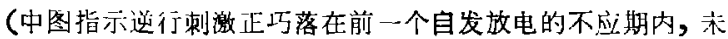
能产生逆行激活反应; 上和下图均表示证行激活反应)

\section{2. 借助传导速度以鉴别 5-HT 神经元}

本项实验设计和 Wessendorf 等人所报道的相同 ${ }^{[7]}$, 只是用了不同种的动物和神经毒. 总

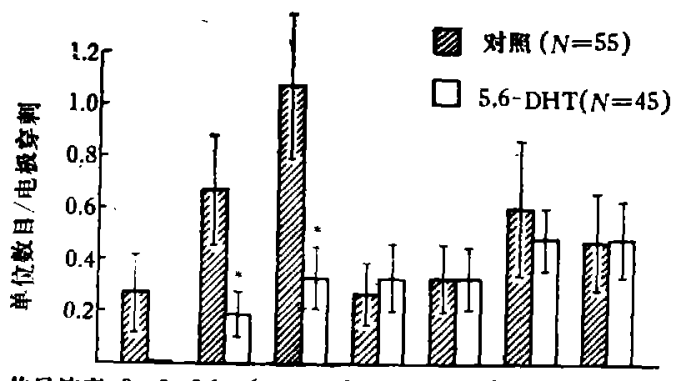

传昌速度 $0-3 \quad 3.1-66.1-12^{12.1}-18 \quad 24.1-30>30 \mathrm{~m} / \mathrm{s}$ 纤维直径 $<1 \sim 1 \quad 1-2 \quad 2-3 \quad 3-4 \quad 4-5>5 \mu \mathrm{m}$

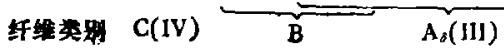

图 3 正常和经 5,6-DHT 处理的两组大鼠中缝大核 㖓脊单位在不同轴突传导速度范围内的分布

(柱高和短直线分别代表均值和标准差， $*$ 表示 $P<0.02$, 纵 坐标指在平均每个电极穿刺针迹内所记到的謎脊单位数, 横 坐标下方标出传导速度所对应的神经纤维直径和类别) 物进行实验,具体方法同正常对照组. 共进行 15 次电极穿刺, 记录到 45 个单位, 然后按不同 10 只正常大鼠上作 21 次电极穿剌, 共记录 到 55 个单位. 它们的轴突传导速度为 $0.8-$ $40 \mathrm{~m} / \mathrm{s}$. 依照不同速度将这些謎脊单位分类 (图 3 斜线方柱), 可以发现较多单位分布在 $3.1-6 \mathrm{~m} / \mathrm{s}$ 和 $6.1-12 \mathrm{~m} / \mathrm{s}$ 两类中. 这样的 分布略有别于以往的报道 ${ }^{[7]}$. 在另一组 10 只大鼠上,事先用 5,6-DHT 外理. 即用戊 巴比妥将动物麻醉, 经侧脑室注射 5,6-DHT (自由硜) $75 \mu \mathrm{g}$, 该药系溶于含 $0.1 \%$ 抗坏血 酸的 $20 \mu \mathrm{l}$ 溶液内. 根据术后最初几天动物 的行为变化，诸如：多动、攻击、不眠、少饮 等, 以及死后脑室内残留棕色药液来判别药 物处理的效果. 给药 7 至 14 天后, 取这些动 的看来，本文资料和他们的报告相一致. 在 
的传导速度作频次分布. 图 3 比较了两组实验结果. 给药组在 3 个传导速度类别的单位数目 明显地减少. 有趣的是在 $0-3 \mathrm{~m} / \mathrm{s}$ 类别, 没有找到逆行激活的单位, 提示含 $5-\mathrm{HT}$ 的缝脊 神经元轴突是无髓鞘的. 此外, 在 3.1-6 m/s 和 $6.1-12 \mathrm{~m} / \mathrm{s}$ 两类中, 琎脊单位数目也明显 下降 $(P<0.02$, 双尾 $\mathrm{t}$ 测验), 提示有些细的有髓鞘轴突的缝脊神经元也是 $5-\mathrm{HT}$ 的. 这些 资料和以往苂光组化和电子显微镜的观察是一致的 ${ }^{[10-12]}$. 至于其它传导速度类别, 对照和给 药两组间无甚差别。

\section{3. 中绕大核 5-HT 神经元的自发放电}

在中㖓大核区域内, 逆行激活的㖓脊神经元接近 $40 \%$ 是没有自发活动的, 其余的自发放 电率差别也很大. 有些单位放电慢至低于 $1 \mathrm{~Hz}$, 而另一些则快达 $60 \mathrm{~Hz}$. 图 4 显示自发放电率 和传导速度之间没有明显的相关性. 然而,比较对照和给药两组的全部资料,可以发现在由整 线为界的方形区内, 仅有的 7 个单位都是从正常动物组记录到的. 换言之,经 5,6-DHT 处理 的动物不再有传导速度慢于 $12 \mathrm{~m} / \mathrm{s}$ 而自发放电率又低于 $3 \mathrm{~Hz}$ 的单位活动. 据此, 可以认为 用上述两项电生理学指标，有可能在生理实验条件下鉴别出中缝大核内的 5-HT 神经元. 此 外,将图 4 各单位的记录点描绘在解剖简图上 (图 5), 结果表明所鉴定的 5-HT 神经元多数 位于中经大核尾部,并混杂于非 5-HT 神经元之中.

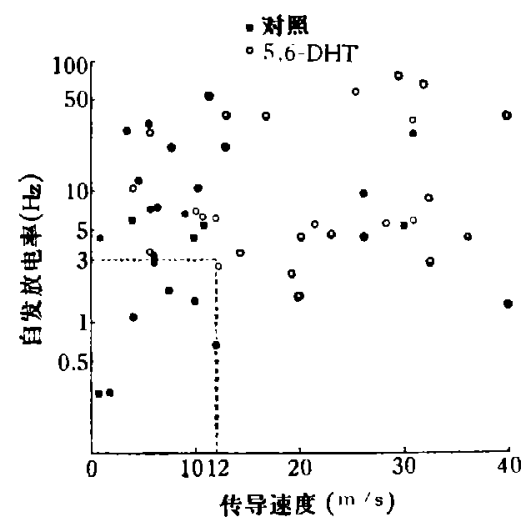

图 4 正常和经 5,6-DHT 处理的两组大鼠 中缝大核缝脊单位的自发放电率对传导速 度的关系

（注意正常组有 7 个单位位于由虚线构成 的区域内, 由此被推测为 5-H'T 神经元)

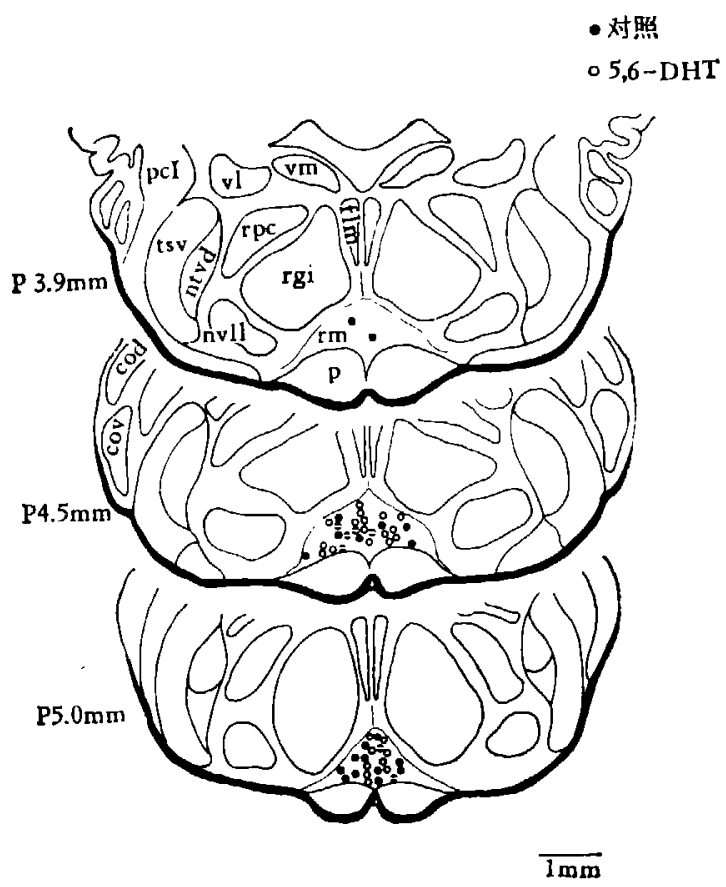

图 5 延桥脑横切面简图, 展示中謎大核内有自 发活动的嗹脊单位的记录点

（黑点和空圈分別代表从正常和经 5,6-DHT 处理的两 组动物上所记到的单位, 有白色横线的黑点则代表图 4 虚线区域内的单位)

有些文献报道,中㖓背核 5-HT 神经元的自发放电很慢 $(0.25--2 \mathrm{~Hz})$, 且很规则 ${ }^{[13-15]}$. 是 否中琏大核 5-HT 神经元的放电也是如此呢? 为了检验这个问题,本实验选取 $2-10 \mathrm{~min}$ 的 


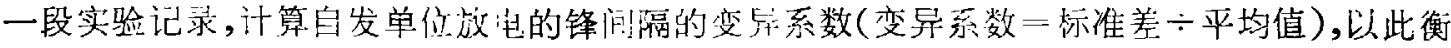

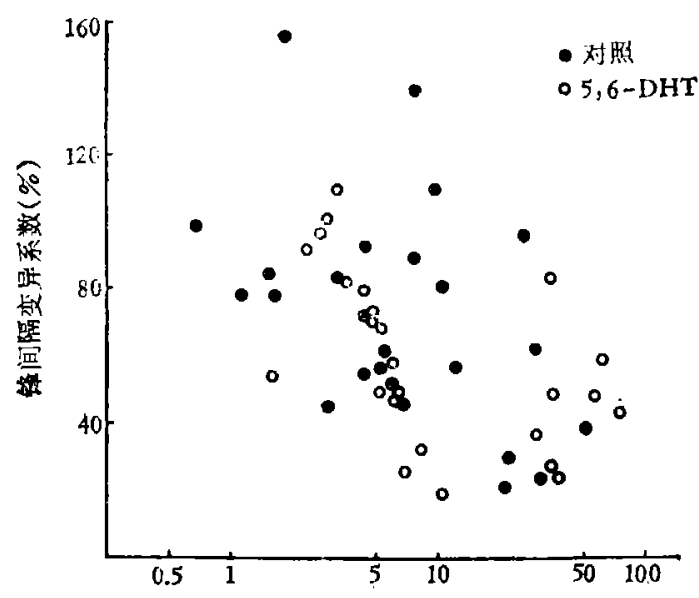

自发故电率 $(\mathrm{Hz})$

图 6 玨常和经 5, 6-DHT 处理的两组大鼠琏脊单位 自发放电率和锋间隔的变异系数之间的相关关系 笔放电的规则性。如图 6 所示, 以刘照和给 药两组各 25 个单位作图,比较它们放电规则 性和传导速度问的关系. 结果出乎所料, 传 导速度快的单位放电比传导速度慢的更规则 些. 这就告诉我们, 需要对规则性作准确测 量, 因为在实验过程你, 那些传导速度慢的单 位放电通过监听器喇叭传放出来, 给人以十 分规则的印象. 这里还需指出, 经 5,6-DHT 处理的动物, 其单位放电在节律方面无异于 正常组. 因此,如 Nakahama 所报道的,中缝 背核 5-HT 神经元放电有好的规律性 ${ }^{[15]}$, 我 们在中缝大核的实验研究中未见.

\section{三、讨 论}

始于七十年代初期, 5, 6-DHT 作为一 种专一的神经毒, 已被广泛用来损毁 5-HT 神经元. 但由于其毒性甚大，以后常被 5,7DHT 所替代 ${ }^{[16]}$. 本实验室倾向于用 5,6-DHT, 因为它对 5-HT 神经元具有高度选择性, 这点显然优于 5,7-DHT. 我们所用脑室内注射 $75 \mu \mathrm{g}$ 的剂量, 就是参照以往的工作经验 ${ }^{[2]}$.

过去的荧光组化研究报道, 5-HT 神经元的下行轴突是无髓鞘的, 并且胞体多半位于中缝 大核尾半部 ${ }^{[6,11,17]}$, 这和本项实验的组织学检查结果相符. 虽然还有一些研究报道, 㖓脊神经 元胞体可延伸到大核的咀部,可是这些学者并没有鉴别这些神经元是否含有 $5-\mathrm{HT}^{[18,19]}$ ?

曾有两篇文章指出, 猫的琏脊单位的轴突传导速度为 $2-5 \mathrm{~m} / \mathrm{s}$, 相当于细的有䯣鞘 纤 维 ${ }^{[9,20]}$. 在大鼠 Wang 和 Aghajanian 发现中琏背核 5-HT 神经元的轴突传导速度为 $0.5-$ $1.7 \mathrm{~m} / \mathrm{s}^{[21]}$, 而Wessendorf 等人最近报道, 中㖓大核的 $5-\mathrm{HT}$ 单位则有 $0.7-1 \mathrm{~m} / \mathrm{s}$ 和 3.1 $6 \mathrm{~m} / \mathrm{s}$ 两类速度, 分别属于无髓鞘和细的有髓鞘两类纤维 ${ }^{[7]}$. 对脊髓背角胶质区的电子显微镜 研究表明,含 5-HT 的末梢是来源于下行的无䯣鞘和细的有髓鞘纤维 ${ }^{\left[{ }^{[12}\right]}$. 本项实验用上海饲 养的 Wistar 大鼠所做的结果, 大体和 Wessendorf 等人的吻合. 差别仅在 $0-3 \mathrm{~m} / \mathrm{s}$ 速度范围 内未能找到较多的缝脊单位, 鉴于本实验采用很强的逆行刺激电流 (达 $9 \mathrm{~mA}$ ), 并经常记录到 振幅相当大的锋电位, 故不像是实验技术的不足才造成上述结果, 相反, 很可能是由于两个实 验室分别用了不同种系的动物才产生上述矛盾. 关于动物种系对研究 5-HT 神经元活动的 重要性,已为前人所强调[?].

本项研究结果提示, 在乌拉坦麻醉下, 自发的单位放电速率可被用来区分中缝大核内的 5-HT 与非 5-HT 两类神经元. 如图 4 所示, 在虚线勾描的区域内找不着给药组动物的逆行 激活单位放电. 结合对琏脊神经元轴突传导速度分析的结果，可以作出假设：中缝大核 5HT 神经元不仅其轴突传导慢于 $12 \mathrm{~m} / \mathrm{s}$, 而且其自发放电率低于 $3 \mathrm{~Hz}$. 以往有大量工作表明, 麻醉大鼠中缝背核的 5-HT 神经元的自发放电率低于 $2 \mathrm{~Hz}^{\left[1^{3-15]}\right.}$. 特别有趣的是我们最近的 工作还表明，用这两个指标鉴定的 $5-\mathrm{HT}$ 单位放电可以被注射麦角副酸二乙酰胺 (LSD) 所 
阻抑, 而那些非 5-HT 单位则不受影响. 这和以往在中㖓背核处所作的观察一致 ${ }^{[22]}$.

曾有不少工作指出, 乌拉坦麻醉大鼠的中㖓背核 5-HT 神经元的自发放电很慢, 且十分 规则 ${ }^{[13,15]}$. 这和本项实验在中琏大核上所见不同. 用锋电位间隔的变异系数作为指标, 我们 发现中缝大核内的㖓脊神经元,包括 5-HT 神经元在内,其自发放电越快,便越有规则性. 这 个差别可能暗示这两个核团的功能有所不同. 看来, 需要对中琏大核㖓冷神经元的放电型式 作进一步的研究.

\section{考文 献}

[1] Basbaum, A. I. and Fields, H. L., Ann. Neniul., 4(1978), 451.

[2] Chiang, C. Y. et al., Chin. Med. J., 92(1979), 129.

[ 3 ] Dickenson, A. H. et al., Brain Research, 170 (1979), 95.

[ 4] Oliveras, J. L. et al., ibid., 164(1979), $3: 7$.

[ 5 ] Proudfit, H. K. et al., ibid., 98(1975), 612.

[ 6 ] Wilklund, L. et al., J. comp. Neurol, 203(1981), 613.

[ 7 ] Wessendorf, M. W. et al., Brain Research, 214(1981), 168.

[ 8 ] Palkovits, M. and Jacobowitz, D. M., J. comp. Neurol., 157(1974), 29.

[ 9 ] Wolstencroft, J. Hi., J. Physiol. (London), 174(1964), 91.

[10] Dahlström, A. and Fuxe, K., Acta Physiol. Scand., 62, Suppl. 232(1964), 1.

[11] — ibid., 64, Suppl. 247(1965), 1.

[12] Ruda, M. A. and Gobel, S., Brain Research, 184(1980), 57.

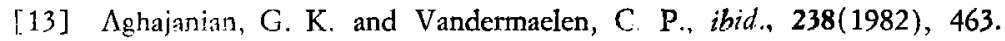

[14] Mosko, S. S. and Jacobs, B. L., Physiol. Behav., 13(1974), 589.

[15] Nakahama, H. et al., Neurosc. Lett., 23(1981), 161.

[16] Cooper, J. R. et al., The Biochemical Basis of Neuropharmacology, 4th ed., Oxford Univ. Press; New York, 1982, 240.

[1.7] Bowker, R. M. et al., Brain Research, 226(1981), 187.

[18] Leichnetz, G. R. et al., Neurosc. Lett., 8(1978), 119.

[19] Zemlan, F. P. et al., J. Anat., 128(1979), 499.

[20] West, D. C. and Wolstencroft, J. H., Nettrosc. Lelt., 5(1977), 147.

[21] Wang, R. Y. and Aghajanian, G. K., Brain Research, 132(1977), 186.

[22] Aghajanian, G. K. et al., Science, 161(1968), 706. 